\title{
Descriptive epidemiology of birth trauma in the United States in 2003
}

\author{
Erin K. Sauber-Schatz ${ }^{a}$ Nina Markovic ${ }^{a}$, Harold B. Weiss ${ }^{a}$, Lisa M. Bodnar ${ }^{a}$, John W. Wilson ${ }^{b}$ and Mark D. Pearlman ${ }^{c}$ \\ Departments of ${ }^{a}$ Epidemiology and ${ }^{b}$ Biostatistics, University of Pittsburgh, Graduate School of Public Health, Pittsburgh, PA, and \\ 'Department of Obstetrics and Gynecology and Department of Surgery, University of Michigan, Ann Arbor, MI, USA
}

Correspondence:
Erin K. Sauber-Schatz, PhD,
MPH, Florida Department of
Health, Division of Family
Health Services, MCH
Practice and Analysis Unit,
4052 Bald Cypress Way Bin
A13, Tallahassee, FL
32399-1721, USA.
E-mail:
Erin_Sauber-Schatz@doh.state.
fl.us

\section{Summary}

Sauber-Schatz EK, Markovic N, Weiss HB, Bodnar LM, Wilson JW, Pearlman MD. Descriptive epidemiology of birth trauma in the United States in 2003. Paediatric and Perinatal Epidemiology 2010; 24: 116-124.

The rate of birth trauma in the US has been reported to range between 0.2 and 37 birth traumas per 1000 births. Because of the minimal number of population-based studies and the inconsistencies among the published birth trauma rates, the rate of birth trauma in the US remains unclear. This is a cross-sectional study that was conducted using 890582 in-hospital birth discharges from the 2003 Healthcare Cost and Utilization Project Kids' Inpatient Database. A neonate was defined as having birth trauma if their hospital discharge record contained an International Classification of Diseases, Ninth Revision, Clinical Modification diagnosis code from 767.0 to 767.9. Weighted data were used to calculate rates for all birth traumas and specific types of birth traumas, and rates and odds ratios by demographic, hospital and clinical variables. Weighted data represented a national estimate of 3920787 in-hospital births.

Birth trauma was estimated to occur in 29 per 1000 births. The three most frequently diagnosed birth traumas were injuries to the scalp, other injuries to the skeleton and fracture of the clavicle. Significant univariable predictors for birth trauma included male gender, Asian or Pacific Islander race, living in urban or wealthy areas, being born in Western, urban and/or teaching hospital, a co-diagnosis of high birthweight, instrument delivery, malpresentation and other complications during labour and delivery. Birth trauma risk factors including those identified in this study may be useful to consider during labour and delivery. In conclusion, additional research is necessary to identify ways to reduce birth trauma and subsequent infant morbidity and mortality.

Keywords: birth trauma, fractured clavicle, birth prevalence, birthweight, mode of delivery, ethnic origin, urban areas, neighbourhood income, teaching hospital.

\section{Introduction}

Birth trauma is defined as an injury sustained by the neonate during the process of labour and delivery. ${ }^{1-3}$ It usually results from trauma sustained during a difficult delivery or secondary to obstetric manipulation of the fetus to allow for delivery., The incidence of birth trauma has reportedly decreased over time because of improvements in obstetric care and prenatal diagnosis; ${ }^{5}$ however, it still occurs even in the presence of highly skilled obstetric and neonatal care. ${ }^{3}$

In studies conducted primarily at single hospitals, birth trauma has been estimated to occur in $2-7 \%$ of all deliveries and is associated with an increased risk of infant morbidity and mortality. ${ }^{3,6,7}$ The few studies that provide population-based national birth trauma estimates report rates ranging from 0.2 to 37 birth traumas per 1000 births..$^{8-10}$ The various birth trauma definitions, study populations and methods used throughout the birth trauma literature make comparisons among or meta-analyses of studies difficult. Because of the minimal number of population-based studies and the inconsistencies among the published birth trauma rates, the rate of birth trauma in the US remains unclear. 
The purpose of this study was to determine the rate of birth trauma in the US through the utilisation of a population-based sample of in-hospital births. The specific aims were: (i) to determine a national estimate of the rate of birth trauma, (ii) to determine the rates of specific types of birth trauma, and (iii) to report the rates and odds ratios (OR) of birth trauma stratified by demographic, hospital and various clinical variables.

\section{Methods}

The data source for this study was the 2003 Healthcare Cost and Utilization Project (HCUP) Kids' Inpatient Database (KID). The KID is an ongoing part of HCUP sponsored by the Agency for Healthcare Research and Quality (AHRQ); it is the only database on children's hospital use, outcomes and charges in the US. ${ }^{11}$ The 2003 KID collected hospital discharge data from 3438 community, non-rehabilitation hospitals in 36 states (AZ, CA, CO, CT, FL, GA, HI, IA, IL, IN, KS, KY, MD, MA, MI, MN, MO, NC, NE, NH, NJ, NV, NY, OH, OR, RI, SC, SD, TN, TX, UT, VA, VT, WA, WI and WV) for the year 2003. ${ }^{11}$ Hospital discharges from federal hospitals (Veterans Administration, Department of Defense and Indian Health Service hospitals), longterm hospitals, psychiatric hospitals, alcohol/chemical dependency treatment facilities and hospital units within institutions (such as prisons) are excluded from the KID. ${ }^{11}$ The KID is described in further detail elsewhere. ${ }^{12}$
Only births in hospital were included in this study. These births were identified in KID as a record with a principal or secondary diagnosis code ranging between V3000 and V3901 (with the last two digits of ' 00 ' or ' 01 ') and where the patient was not transferred from another facility. ${ }^{11}$ After this exclusion, the study sample consisted of 890582 in-hospital birth discharges from the 2003 KID. Discharge weights were applied to the study sample to adjust the data to represent nationwide birth discharges and obtain national estimates. The KID discharge weights were developed using the American Hospital Association universe as the standard and by post-stratification of hospitals on ownership/control, bed size, teaching status, rural/ urban location, geographical region and hospital type (children's hospital or other). ${ }^{11}$ After applying the weights, the data represented a weighted national estimate of 3920787 in-hospital birth discharges for 2003.

Birth trauma was defined in this study as an International Classification of Diseases, Ninth Revision, Clinical Modification (ICD-9-CM) diagnosis code from 767.0 to 767.9 in any one of the 15 diagnosis variables in the KID. The ICD-9-CM was 'the official system of assigning codes to diagnoses and procedures associated with hospital utilisation in the United States' in $2003 .{ }^{13}$ Birth trauma was further classified by specific type of birth trauma, including subdural and cerebral haemorrhage (ICD-9-CM 767.0), injuries to the scalp (ICD-9-CM 767.1), fracture of the clavicle (ICD-9-CM 767.2), other injuries to the skeleton (ICD-9-CM 767.3),

Table 1. Number and rate per 1000 in-hospital births of reported birth trauma in the US, 2003 by type of birth trauma

\begin{tabular}{|c|c|c|c|c|}
\hline Type of birth trauma (ICD-9 codes) & Un-weighted $n$ & $\begin{array}{l}\text { Weighted } \\
\text { estimate }\end{array}$ & $\begin{array}{l}\text { SD of weighted } \\
\text { estimate }\end{array}$ & $\begin{array}{c}{ }^{a} \text { Weighted rate per } \\
1000 \text { births }\end{array}$ \\
\hline All birth trauma (767.0-767.9) & 44658 & 111989 & 3227 & 28.56 \\
\hline Injuries to the scalp (767.1) & 22764 & 78644 & 2487 & 20.06 \\
\hline Other injuries to the skeleton (767.3) & 9525 & 14499 & 1160 & 3.70 \\
\hline Fracture of the clavicle (767.2) & 6353 & 9545 & 300.10 & 2.43 \\
\hline Other specified birth trauma (767.8) & 3994 & 6136 & 289.94 & 1.56 \\
\hline Injury to the brachial plexus (767.6) & 3302 & 5021 & 143.06 & 1.28 \\
\hline Subdural and cerebral haemorrhage (767.0) & 1064 & 1599 & 77.49 & 0.41 \\
\hline Facial nerve injury (767.5) & 661 & 1014 & 50.69 & 0.26 \\
\hline Birth trauma, unspecified (767.9) & 218 & 339 & 32.25 & 0.09 \\
\hline Other cranial and peripheral nerve injuries (767.7) & 124 & 192 & 18.39 & 0.05 \\
\hline Injury to the spine and spinal cord (767.4) & 10 & 15 & 4.79 & 0.00 \\
\hline Total number of births & 890582 & 3920787 & 61581 & \\
\hline
\end{tabular}

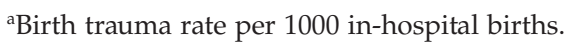

ICD-9, International Classification of Diseases, Ninth Revision; SD, standard deviation. 
Table 2. Univariable analysis of infants diagnosed with birth trauma by demographic variables (weighted), Kids' Inpatient Database 2003

\begin{tabular}{|c|c|c|c|c|c|c|c|}
\hline Demographic variables & $n_{\mathrm{WE}}$ & $n_{\mathrm{BT}}$ & $\begin{array}{c}{ }^{a} \text { Rate per } \\
1000\end{array}$ & OR & {$[95 \% \mathrm{CI}]$} & $P$-value & $\%$ missing \\
\hline Infant gender & & & & & & $<0.001$ & 0.6 \\
\hline Female & 1900219 & 47439 & 24.97 & 1.00 & Reference & & \\
\hline Male & 1997286 & 63996 & 32.04 & 1.29 & {$[1.25,1.34]$} & & \\
\hline Race & & & & & & $<0.001$ & 28.4 \\
\hline White & 1486381 & 41877 & 28.17 & 1.00 & Reference & & \\
\hline Hispanic & 665940 & 19240 & 28.89 & 1.03 & {$[0.93,1.14]$} & & \\
\hline Black & 354448 & 8888 & 25.08 & 0.89 & {$[0.81,0.98]$} & & \\
\hline Other & 169286 & 4736 & 27.98 & 0.99 & {$[0.88,1.12]$} & & \\
\hline Asian or Pacific Islander & 118592 & 4692 & 39.56 & 1.42 & {$[1.25,1.62]$} & & \\
\hline Native American & 13178 & 249 & 18.90 & 0.67 & {$[0.48,0.92]$} & & \\
\hline Birthweight & & & & & & $<0.001$ & 78.1 \\
\hline Low $(<2500 \mathrm{~g})$ & 76990 & 1300 & 16.89 & 0.54 & {$[0.47,0.62]$} & & \\
\hline Normal (2500-3999 g) & 702695 & 21534 & 30.65 & 1.00 & Reference & & \\
\hline High $(\geq 4000 \mathrm{~g})$ & 78952 & 3710 & 46.99 & 1.56 & {$[1.41,1.72]$} & & \\
\hline Short gestation; low birthweight; fetal growth retardation ${ }^{\mathrm{b}}$ & & & & & & $<0.001$ & 0.0 \\
\hline Yes & 448673 & 10486 & 23.37 & 0.80 & {$[0.72,0.88]$} & & \\
\hline No & 3472114 & 101504 & 29.23 & 1.00 & Reference & & \\
\hline Location of the patient & & & & & & $<0.001$ & 0.3 \\
\hline Large metropolitan & 2279144 & 71524 & 31.38 & 1.00 & Reference & & \\
\hline Small metropolitan & 1070318 & 26750 & 24.99 & 0.79 & {$[0.71,0.88]$} & & \\
\hline Micropolitan & 347542 & 8778 & 25.30 & 0.80 & {$[0.71,0.90]$} & & \\
\hline Non-core & 210552 & 4571 & 21.71 & 0.69 & {$[0.59,0.79]$} & & \\
\hline Median household income for patient's zip code & & & & & & $<0.001$ & 1.6 \\
\hline$\$ 60000+$ & 931933 & 29185 & 31.32 & 1.00 & Reference & & \\
\hline$\$ 45000-59999$ & 989247 & 29366 & 29.69 & 0.95 & {$[0.88,1.02]$} & & \\
\hline$\$ 36000-44999$ & 977151 & 26550 & 27.17 & 0.86 & {$[0.79,0.94]$} & & \\
\hline$\$ 1-35999$ & 958347 & 24978 & 26.06 & 0.83 & {$[0.75,0.92]$} & & \\
\hline Payer information & & & & & & $<0.001$ & 0.1 \\
\hline Private (including HMO) & 2092341 & 60531 & 28.93 & 1.00 & Reference & & \\
\hline Medicaid & 1515240 & 43123 & 28.46 & 0.98 & {$[0.92,1.05]$} & & \\
\hline Self-pay & 187648 & 5074 & 27.04 & 0.93 & {$[0.83,1.05]$} & & \\
\hline Other & 109885 & 2853 & 25.96 & 0.90 & {$[0.72,1.12]$} & & \\
\hline No charge & 5521 & 210 & 38.04 & 1.33 & {$[0.88,2.00]$} & & \\
\hline Medicare & 4917 & 100 & 20.33 & 0.70 & {$[0.49,0.995]$} & & \\
\hline
\end{tabular}

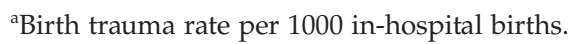

${ }^{\mathrm{b} C}$ ategory from Clinical Classification Software.

$\mathrm{CI}$, confidence interval; $n_{\mathrm{BT}}$, weighted estimate of the number of infants with reported birth trauma; HMO, health maintenance organisation; $n_{\mathrm{WE}}$, weighted estimate of the total number of births by stratum; OR, odds ratio.

injury to the spine and spinal cord (ICD-9-CM 767.4), facial nerve injury (ICD-9-CM 767.5), injury to the brachial plexus (ICD-9-CM 767.6), other cranial and peripheral nerve injuries (ICD-9-CM 767.7), other specified birth trauma (ICD-9-CM 767.8) and birth trauma, unspecified (ICD-9-CM 767.9). This study was approved by the University of Pittsburgh's Institutional Review Board.

All analyses were conducted on the weighted data using SPSS 15.0 (SPSS Inc., Chicago, IL, USA) and SAS version 9.1 (SAS Institute Inc., Cary, NC, USA). SAS's
PROC SURVEYFREQ ${ }^{14}$ procedure was used to determine the national estimates of all birth trauma and specific types of birth trauma. These estimates were then used to calculate the rates of all birth trauma and specific types of birth trauma. SAS's PROC SURVEYLOGISTIC ${ }^{15,16}$ procedure with the total number of primary sampling units in the study population to compute a finite population correction for Taylor series variance estimation was used to calculate ORs, 95\% confidence intervals $[95 \% \mathrm{CI}]$ and $P$-values for several demographic, hospital and clinical variables. The 
Table 3. Univariable analyses of birth trauma by hospital variables (weighted), Kids' Inpatient Database 2003

\begin{tabular}{|c|c|c|c|c|c|c|c|}
\hline Hospital variables & $n_{\mathrm{WE}}$ & $n_{\mathrm{BT}}$ & a'Rate per 1000 & OR & {$[95 \% \mathrm{CI}]$} & $P$-value & $\%$ missing \\
\hline Region of hospital & & & & & & $<0.001$ & 0.0 \\
\hline South & 1446837 & 39283 & 27.15 & 1.00 & Reference & & \\
\hline West & 929069 & 29315 & 31.55 & 1.17 & {$[1.02,1.33]$} & & \\
\hline Midwest & 870745 & 24924 & 28.62 & 1.06 & {$[0.92,1.21]$} & & \\
\hline Northeast & 674136 & 18468 & 27.40 & 1.01 & {$[0.87,1.17]$} & & \\
\hline Hospital bed size & & & & & & $<0.001$ & 0.6 \\
\hline Large & 2463598 & 69758 & 28.32 & 1.00 & Reference & & \\
\hline Medium & 1031358 & 30418 & 29.49 & 1.04 & {$[0.93,1.17]$} & & \\
\hline Small & 400879 & 11296 & 28.18 & 1.00 & {$[0.86,1.15]$} & & \\
\hline Location of hospital & & & & & & $<0.001$ & 0.6 \\
\hline Rural & 524470 & 12315 & 23.48 & 1.00 & Reference & & \\
\hline Urban & 3371364 & 99157 & 29.41 & 1.26 & {$[1.12,1.42]$} & & \\
\hline Teaching status of hospital & & & & & & 0.002 & 0.6 \\
\hline Non-teaching & 2190842 & 58352 & 26.64 & 1.00 & Reference & & \\
\hline Teaching & 1704992 & 53120 & 31.16 & 1.18 & {$[1.06,1.30]$} & & \\
\hline Location \& teaching status of hospital & & & & & & $<0.001$ & 0.6 \\
\hline Rural & 524470 & 12315 & 23.48 & 1.00 & Reference & & \\
\hline Urban non-teaching & 1711037 & 47273 & 27.63 & 1.18 & {$[1.05,1.33]$} & & \\
\hline Urban teaching & 1660328 & 51884 & 31.25 & 1.34 & {$[1.17,1.54]$} & & \\
\hline
\end{tabular}

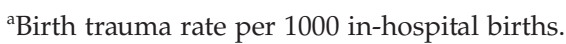

$\mathrm{CI}$, confidence interval; $n_{\mathrm{BT}}$, weighted estimate of the number of infants with reported birth trauma; $n_{\mathrm{WE}}$, weighted estimate of the total number of births by stratum; OR, odds ratio.

STRATA, CLUSTER and WEIGHT statements were also used to specify sample design information in the PROC SURVEYLOGISTIC procedure and to take into account KID's sampling design.

\section{Results}

All of the following results are based on the weighted data. The rate of reported birth trauma was 28.6 or approximately 29 per 1000 in-hospital births (Table 1). This rate extrapolates to a birth trauma diagnosis in approximately 111989 in-hospital births for the US in 2003. Injuries to the scalp were the primary type of birth trauma (20.06 per 1000 births) followed by other injuries to the skeleton (3.70 per 1000 births) and fracture of the clavicle (2.43 per 1000 births) (Table 1).

When the data were stratified by demographic variables (Table 2) it was clear that boys, Asian or Pacific Islanders and high-birthweight infants had higher rates and ORs of reported birth trauma. When the data were stratified by location of the mother (based on an urban to rural designation for the mother's county of residence) the rate and OR of birth trauma decreased as the location became more rural. Similarly the rate and OR of birth trauma decreased as the median household income for the patient's zip code also decreased (Table 2). When the data were stratified by payer information, only those with Medicare had a significantly reduced OR of reported birth trauma compared with those with private payer (Table 2).

The 2003 KID includes 36 of the 50 states. The average rate of birth trauma was 27.2 per 1000 births across all 36 participating states, but the rate ranged from 15.3 cases of reported birth trauma in South Carolina to 57.5 cases of reported birth trauma in Maryland per 1000 in-hospital births. When the data were stratified by various hospital variables, it can be seen that hospitals in the Western United States as well as urban, teaching and urban teaching hospitals reported the highest rates and ORs of birth trauma (Table 3). There were no significant differences in the rates or ORs of birth trauma among hospitals with different bed sizes (Table 3).

Of the infants diagnosed with birth trauma $6.8 \%$ were also diagnosed with a complication of labour and delivery; only $0.9 \%$ of the infants not diagnosed with birth trauma were diagnosed with a complication of labour and delivery (data not shown). The corresponding OR for any complication of labour and delivery for all birth trauma was highly significant $(\mathrm{OR}=7.93,95 \%$ 


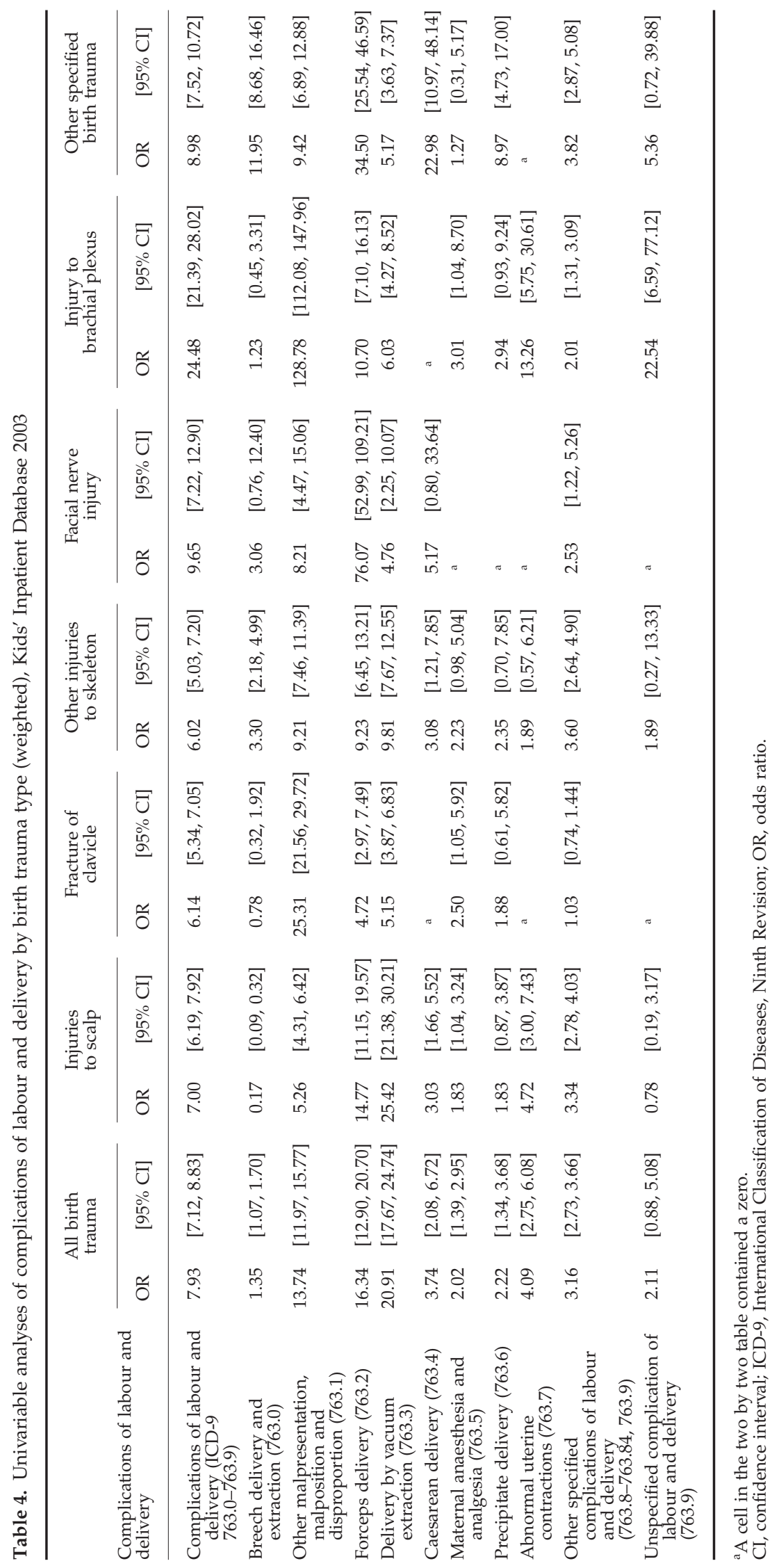


Table 5. Birth trauma rate and univariable analysis of infants diagnosed with birth trauma by severity indicators (weighted), Kids' Inpatient Database 2003

\begin{tabular}{|c|c|c|c|c|c|c|c|}
\hline Severity indicators & $n_{\mathrm{EW}}$ & $n_{\mathrm{BT}}$ & ${ }^{a}$ Rate per 1000 & OR & {$[95 \% \mathrm{CI}]$} & $P$-value & $\%$ missing \\
\hline Length of stay (days) & & & & & & $<0.001$ & 0.0 \\
\hline 0 & 50024 & 801 & 16.01 & 0.50 & {$[0.43,0.57]$} & & \\
\hline 1 & 695598 & 15750 & 22.64 & 0.71 & {$[0.66,0.76]$} & & \\
\hline 2 & 2010960 & 63801 & 31.73 & 1.00 & Reference & & \\
\hline 3 & 641915 & 15665 & 24.40 & 0.76 & {$[0.72,0.81]$} & & \\
\hline 4 & 247479 & 6985 & 28.23 & 0.89 & {$[0.82,0.96]$} & & \\
\hline $5+$ & 274811 & 8988 & 32.71 & 1.03 & {$[0.97,1.10]$} & & \\
\hline Number of diagnoses & & & & & & $<0.001$ & 0.0 \\
\hline 1 & 1577566 & 0 & 0.00 & $\mathrm{~b}$ & & & \\
\hline 2 & 1182679 & 29221 & 24.71 & 1.00 & Reference & & \\
\hline 3 & 562739 & 34060 & 60.53 & 2.54 & {$[2.36,2.74]$} & & \\
\hline 4 & 252729 & 22292 & 88.21 & 3.82 & {$[3.52,4.15]$} & & \\
\hline 5 & 122926 & 11602 & 94.38 & 4.11 & {$[3.76,4.51]$} & & \\
\hline $6+$ & 222149 & 14813 & 66.68 & 2.82 & {$[2.56,3.11]$} & & \\
\hline Number of procedures & & & & & & $<0.001$ & 0.0 \\
\hline 0 & 1929510 & 49731 & 25.77 & 1.00 & Reference & & \\
\hline 1 & 1418424 & 43101 & 30.39 & 1.19 & {$[1.11,1.26]$} & & \\
\hline 2 & 403266 & 13446 & 33.34 & 1.30 & {$[1.17,1.45]$} & & \\
\hline $3+$ & 169587 & 5712 & 33.68 & 1.32 & {$[1.19,1.46]$} & & \\
\hline Total charges & & & & & & $<0.001$ & 3.3 \\
\hline$\$ 0-1093$ & 949224 & 22433 & 23.63 & 1.00 & Reference & & \\
\hline$\$ 1094-1602$ & 948272 & 26352 & 27.79 & 1.18 & {$[1.08,1.29]$} & & \\
\hline$\$ 1603-2584$ & 946681 & 28040 & 29.62 & 1.26 & {$[1.14,1.39]$} & & \\
\hline$\$ 2585+$ & 947877 & 32077 & 33.84 & 1.45 & {$[1.30,1.61]$} & & \\
\hline Died during hospitalisation & & & & & & $<0.001$ & 0.0 \\
\hline No & 3907761 & 111752 & 28.60 & 1.00 & Reference & & \\
\hline Yes & 12306 & 210 & 17.07 & 0.59 & {$[0.49,0.70]$} & & \\
\hline
\end{tabular}

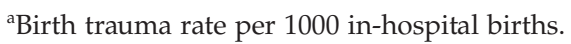

${ }^{\mathrm{b}} \mathrm{A}$ cell in the two by two table contained a zero (a cell).

$\mathrm{CI}$, confidence interval; $n_{\mathrm{BT}}$, weighted estimate of the number of infants with reported birth trauma; $n_{\mathrm{WE}}$, weighted estimate of the total number of births by stratum; OR, odds ratio.

CI [7.12, 8.83], Table 4). The most frequently diagnosed complications of labour and delivery for infants with reported birth trauma were delivery by vacuum extraction $(2.6 \%)$ followed by other malpresentation, malposition and disproportion (2.0\%) and forceps delivery $(0.9 \%)$ (Table 4 ); in comparison, only $0.1 \%$ of infants without birth trauma were diagnosed with each of the above complications (data not shown). These three complications also had the highest unadjusted ORs for all the birth trauma categories (Table 4). The diagnosis of a complication of labour and delivery was also highly significant for each of the specific types of birth trauma (Table 4). However, the prevalence of each complication of labour and delivery varied to some degree by the type of birth trauma reported (Table 4).

When the data were stratified by various severity indicators (Table 5) it was found that the rate of reported birth trauma was highest in neonates with a length of stay $\geq 5$ days, the rate and OR of reported birth trauma was highest in those with five diagnoses and three or more procedures, and as the total hospital charges increased so did the rate and OR of reported birth trauma (Table 5). Interestingly, the rate of birth trauma was higher in the infants that did not die during hospitalisation than in those who died during hospitalisation. Compared with neonates who did not die during hospitalisation, the ones who died were $41 \%$ less likely to have a reported birth trauma (Table 5).

\section{Discussion}

This study's reported birth trauma rate of 28.6 per 1000 births is higher than many other published rates, but is 
similar to the rate reported in Tomashek et al. for the years $1999-2000,{ }^{8}$ which is one of the few studies in the literature that provided a recent national estimate of birth trauma. Their national estimates were derived from the National Hospital Discharge Survey and included 55210 newborns in 1989-90 and 68678 newborns in 1999-2000. ${ }^{8}$ Tomashek et al. estimated that the rate of birth trauma in all newborns was 37.0 per 1000 newborns in 1989-90 and 29.2 per 1000 newborns in 1999-2000. . A 7-year study conducted in Finland and published in 1990 reported the rate of major birth trauma as 31.6 per 1000 livebirths. ${ }^{17}$

Other studies have reported much lower rates of birth trauma. For instance, Hankins et al. in 2006, through an Ovid Medline literature review restricted to the previous 10 years of literature using the search term 'fetal trauma', estimated that the incidence of significant birth trauma varied from 0.2 to 2 per 1000 births. ${ }^{9}$ At a single hospital in Saudi Arabia, Awari et al. in 2003 determined that birth injuries had an incidence of 6.7 per 1000 livebirths through a retrospective review of the medical records of 31028 consecutive deliveries from January 1986 to December $1996 .^{10}$ The reported rate in the study by Awari et al. is higher than Hankins et al., but much lower than the rate reported in the current study and by Tomashek et al.

Interestingly, this study showed that Asian or Pacific Islanders were more likely to experience birth trauma; however, there were no clear reasons for the increased risk. For instance, only $1.3 \%$ of the Asian or Pacific Islander infants experienced any complications of labour and delivery compared with $1 \%$ in all births. Furthermore, of the Asian or Pacific Islander infants with a reported birthweight, $85.9 \%$ were of normal weight and only $5.7 \%$ had a high birthweight compared with a high birthweight in $9.2 \%$ of all births. Some of the increased risk could be as a result of the fact that Asian or Pacific Islander neonates primarily lived in the wealthiest neighbourhoods $(41.8 \%)$ and were born in urban (95.6\%) and Western hospitals (50\%). Another possibility for the increased risk could be anatomical, including pelvic differences among Asian or Pacific Islander women; however, further research is needed.

It is important to point out that neonates with birth trauma have a reduced rate and OR of death during their hospitalisation. We speculate that in some cases, choices are made or procedures are performed during labour and delivery to protect the health and lives of both the pregnant woman and fetus, even if they increase the risk of birth trauma. For instance, if a fetus is known to be experiencing hypoxia or birth asphyxia, a physician may make the decision to use vacuum extraction or forceps delivery to reduce the risk of potential adverse health effects resulting from a lack of oxygen. Although the instrument delivery may cause birth trauma [the OR for birth trauma in infants diagnosed with intrauterine hypoxia and birth asphyxia was $\mathrm{OR}=2.35,95 \% \mathrm{CI}[2.14,2.58]$ in this study (data not shown)], the benefits of using the instrument to resolve hypoxia/asphyxia will likely outweigh the risks of birth trauma.

Also of interest, the birth trauma rate in 'normal newborns' with uncomplicated in-hospital births was 17.5 birth traumas per 1000 in-hospital births (data not shown). This rate is approximately only 11 fewer cases per 1000 in-hospital births than the overall rate of 29 cases of birth trauma per 1000 in-hospital births. This raises the question of what is an acceptable or normal rate of birth trauma. Even during an uncomplicated natural vaginal delivery the neonate is exposed to several forces as it passes through the birth canal that can cause birth trauma. Therefore, it is not unreasonable to believe that the birth trauma rate will never be zero and that a specific rate, perhaps approximately 18 per 1000 in-hospital births, should be considered an acceptable and normal baseline for birth trauma.

This study has several strengths. Specifically, the KID provides current, quality controlled and reliable national data with a much larger sample size and more recent data than any previously published birth trauma study. The large sample size also allowed for rate calculations of specific types of birth trauma as well as stratification of the data by key variables.

Our study was limited by the fact that not all data elements in the KID are provided by each state. ${ }^{11}$ Therefore, some of the analyses used incomplete data. This has the potential to bias some of the results, but with such a large sample size and through the use of advanced statistical procedures, any resulting biases would be very limited. Another limitation is the possible variation among hospital coding practices. The data in the KID rely solely on hospital discharge data and are therefore only as complete and accurate as the hospital reports allow.

However, the use of hospital discharge data is also a strength of this study. Lydon-Rochelle et al. quantified the accuracy of administrative data (birth certificate data, hospital discharge data and combined birth certificate and hospital discharge data) for reflecting 
actual maternal diagnoses and intrapartum procedural status. ${ }^{18}$ They determined that perinatal epidemiological studies should not rely exclusively on birth certificate data. ${ }^{18}$ This study used hospital discharge data that were shown by Lydon-Rochelle et al. to have generally high true positive fractions or accuracy for detecting maternal diagnoses and intrapartum procedures. ${ }^{18}$ Another potential limitation is that it is unknown whether it is the hospital, the population it serves, or a combination thereof, that is driving the increased risk of birth trauma. Further research is needed to explore this issue.

A final limitation is the possibility of confounding. Only diagnoses of birth trauma were taken into account for analyses and, although a majority of birth trauma cases had the same average number of diagnoses as in-hospital births without birth trauma, the potential for confounding because of other diagnoses should be kept in mind when interpreting results.

There are several clinical and public health implications of this study. Through a population-based national estimate it was determined that the rate of birth trauma in the US is higher than a majority of studies have previously reported. Health professionals may have the ability to decrease the number and rate of infants diagnosed with birth trauma by recognising perinatal risk factors for birth trauma and using technological advancements (such as ultrasonography and fetal monitoring) before attempting a vaginal delivery. ${ }^{19}$ In addition, further birth trauma research, including more in-depth classification (such as an expansion of the work done by Pressler ${ }^{19}$ ) and follow-up of infants who are diagnosed with birth trauma, will better quantify the morbidity and mortality of birth trauma by type and among infants and women with various birth trauma risk factors. Prevention of birth trauma will also reduce the number of stresses that it places on the health care system because neonates with birth trauma were shown in this study to have higher costs, greater lengths of stay, and have more medical procedures than neonates not diagnosed with birth trauma. Simply stated, preventing birth trauma will reduce infant morbidity and mortality and reduce the stresses it places on the health care system.

\section{Acknowledgements}

This work resulted as part of Erin Sauber-Schatz's PhD dissertation, and there were no funding sources for this study. The authors would like to thank HCUP for making their data available to researchers.

\section{References}

1 Becerra JE, Fry YW, Rowley DL. Morbidity estimates of conditions originating in the perinatal period: United States, 1986 through 1987. Pediatrics 1991; 88:553-559.

2 Towner D, Castro MA, Eby-Wilkens E, Gilbert WM. Effect of mode of delivery in nulliparous women on neonatal intracranial injury. New England Journal of Medicine 1999; 341:1709-1714.

3 Parker LA. Part 1: early recognition and treatment of birth trauma: injuries to the head and face. Advances in Neonatal Care 2005; 5:288-297; quiz 298-300.

4 Gherman RB, Goodwin TM, Ouzounian JG, Miller DA, Paul $\mathrm{RH}$. Brachial plexus palsy associated with cesarean section: an in utero injury? American Journal of Obstetrics and Gynecology 1997; 177:1162-1164.

5 Uhing MR. Management of birth injuries. Pediatric Clinics of North America 2004; 51:1169-1186, xii.

6 Welch K, Strand R. Traumatic parturitional intracranial hemorrhage. Developmental Medicine and Child Neurology 1986; 28:156-164.

7 Menticoglou SM, Perlman M, Manning FA. High cervical spinal cord injury in neonates delivered with forceps: report of 15 cases. Obstetrics and Gynecology 1995; 86(4 Pt 1): 589-594.

8 Tomashek KM, Crouse CJ, Iyasu S, Johnson CH, Flowers LM. A comparison of morbidity rates attributable to conditions originating in the perinatal period among newborns discharged from United States hospitals, 1989-90 and 1999-2000. Paediatric and Perinatal Epidemiology 2006; 20:24-34.

9 Hankins GD, Clark SM, Munn MB. Cesarean section on request at 39 weeks: impact on shoulder dystocia, fetal trauma, neonatal encephalopathy, and intrauterine fetal demise. Seminars in Perinatology 2006; 30:276-287.

10 Awari BH, Al-Habdan I, Sadat-Ali M, Al-Mulhim A. Birth associated trauma. Saudi Medical Journal 2003; 24:672-674.

11 Agency for Healthcare Research and Quality. Introduction to the HCUP KIDs' Inpatient Database (KID) 2003. Rockville, MD: HCUP Central Distributor, 2007.

12 HCUP. KID Database Documentation. Rockville, MD: AHRQ, 2008.

13 National Center for Health Statistics. Classification of Diseases and Functioning, and Disability. Hyattsville, MD: NCHS, 2008. http://www.cdc.gov/nchs/icd9cm.htm [last accessed 18 November 2009].

14 An AB, Watts D. New SAS Procedures for Analysis of Sample Survey Data. Cary, NC: SAS, 2007.

15 An AB. Performing Logistic Regression on Survey Data with the New SURVEYLOGISTIC Procedure. Advanced tutorials. Paper 258. Orlando, FL: SUGI 272002.

16 SAS Institute Inc. SAS/STAT 9.2 User's Guide: The SURVEYLOGISTIC Procedure. Cary, NC: SAS Institute Inc., 2008. 
17 Salonen IS, Uusitalo R. Birth injuries: incidence and predisposing factors. Zeitschrift Für Kinderchirurgie 1990; 45:133-135.

18 Lydon-Rochelle MT, Holt VL, Nelson JC, Cárdena V, Gardella C, Easterling TR, et al. Accuracy of reporting maternal in-hospital diagnoses and intrapartum procedures in Washington State linked birth records. Paediatric and Perinatal Epidemiology 2005; 19:460-471.

19 Pressler JL. Classification of major newborn birth injuries. Journal of Perinatal and Neonatal Nursing 2008; 22:60-67. 